\title{
Subluminous phase velocity of a focused laser beam and vacuum laser acceleration
}

\author{
Y.K. Ho*, X.Q. Yuan, J. Pang, N. Cao, Q. Kong, P.X. Wang, and L. Shao \\ Institute of Modern Physics, Fudan University, Shanghai 200433, China \\ E.H. Esarey and A.M. Sessler \\ Lawrence Berkeley National Laboratory, University of California, CA 94720
}

\begin{abstract}
It has been found that for a focused laser beam propagating in free-space, there exists, surrounding the laser beam axis, a subluminous wave phase velocity region. Relativistic electrons injected into this region can be trapped in the acceleration phase and remain in phase with the laser field for sufficiently long times, thereby receiving considerable energy from the field. Optics placed near the laser focus are not necessary, thus allowing high intensities and large energy gains. Important features of this process are examined via test particle simulations. The resulting energy gains are in agreement with theoretical estimates based on acceleration by the axial laser field.
\end{abstract}

PACS number: 41.75.Jv, 42.60.Jf, 41.85.Ja, 52.38.Kd 
For more than a decade there has been much interest in studying the interactions of ultraintense lasers with matter [1]. Laser acceleration of charged particles has been one of those frontier research subjects [2-5]. Concerning laser acceleration in vacuum, there were many discussions and arguments lasting decades [6-13], and the crucial issue in those discussions is related to wave phase velocity. For example, the so-called LawsonWoodward theorem $[6,7]$ states that a relativistic electron interacting with a focused laser beam in vacuum can not gain energy provided that (i) the electron trajectory is a straight line with a speed $v_{e}$ approximately equal to the speed of light in vacuum, $v_{e} \approx c$, which is unperturbed by the laser field, and (ii) the interaction region is infinite in extent. The essential physics underlying this theorem is that the phase velocity of the laser field near the focal region is greater than $c$. Thus, it was argued that the inevitable phase slippage would lead the relativistic electron to experience alternatively acceleration and deceleration phase regions as it transverses the laser field, which would result in a cancellation of the energy gain for an unlimited interaction length. More recently it was shown, via experiment [8] and simulation [9-11], that a focused laser pulse interacting with low energy electrons (at rest or nearly at rest, and thereby not subject to the LawsonWoodward theorem) could be ponderomotively scattered and receive a net energy gain by interacting with the nonlinear ponderomotive laser force. Simulations of ponderomotive scattering indicate, however, that the energy gain is intrinsically limited to relatively low values, i.e., less than $10 \mathrm{MeV}$ for presently obtainable laser intensities [9-11].

In this Letter we will show that the Lawson-Woodward theorem can be circumvented at high intensities that can be delivered by present laser systems. For a focused laser beam propagating in vacuum, there exists a region characterized by subluminous phase velocity. Based on this feature we are able to propose a novel vacuum laser acceleration 
scheme, the capture and acceleration scenario (CAS) [12, 13]. Previous studies of the CAS found significant energy gains only in the regime of ultra-high intensities $a_{0} \gtrsim 100$ when $k w_{0}>170$, where $a_{0}=e E_{0} /(m \omega c)=8.5 \times 10^{-10} \lambda I^{1 / 2}$ with $\lambda$ the laser wavelength in $\mu \mathrm{m}, I$ the intensity in $\mathrm{W} / \mathrm{cm}^{2}, E_{0}$ the electric field amplitude of the laser beam at focus, $\omega=c k=2 \pi c / \lambda$ the laser frequency, and $w_{0}$ the beam width at focus. In this Letter, a new regime of the CAS is described in which significant energy gains ( $>100$ $\mathrm{MeV})$ can result for presently achievable intensities $\left(a_{0}>10\right)$ and powers $(>100 \mathrm{TW})$. In this regime $\left(5<a_{0}<100\right.$, and $\left.k w_{0}<120\right)$, the energy gain is found to scale linearly in the laser field and is in agreement with theoretical estimates of acceleration by the axial laser field. Simulations indicate that the electron trajectory is significantly perturbed by the laser field as it enters the high intensity channel, which in effect limits the interaction region, thereby circumventing the Lawson-Woodward theorem. Hence, large energy gains are obtained without limiting the interaction distance by the use of additional optics (as required in Ref. [14]). This allows operation at ultra high laser intensities and high energy gains without the restriction of damaging nearby optics.

For a laser beam of Hermite-Gaussian $(0,0)$ mode polarized in the $\mathrm{x}$-direction and propagated along the z-axis, the transverse electric field component is [15]

$$
E_{x}(x, y, z, t)=E_{0} \frac{w_{0}}{w(z)} \exp \left[-\frac{r^{2}}{w(z)^{2}}-i\left(\varphi+\varphi_{0}\right)\right],
$$

where $w(z)=w_{0}\left(1+\alpha^{2}\right)^{1 / 2}$ is the beam width, $\alpha=z / Z_{R}, Z_{R}=k w_{0}^{2} / 2$ is the Rayleigh length, $r^{2}=x^{2}+y^{2}, \varphi_{0}$ is the initial phase, and with phase

$$
\varphi=k z-\omega t-\tan ^{-1} \alpha+\frac{k r^{2}}{2 z\left(1+1 / \alpha^{2}\right)} .
$$

The effective phase velocity of the wave along a particle trajectory, $\left(\mathbf{v}_{\varphi}\right)_{J}$, is given by

$$
\partial \varphi / \partial t+\left(\mathbf{v}_{\varphi}\right)_{J} \cdot(\nabla \varphi)_{J}=0,
$$


where $(\nabla \varphi)_{J}$ is the gradient of the phase field along the trajectory. In particular, the the effective phase velocity along a trajectory parallel to the z-axis is $v_{\varphi z}=c k /(\partial \varphi / \partial z)$, and the minimum phase velocity is $v_{\varphi m}=c k /|\nabla \varphi|$, which occurs at the angle $\theta_{m}$ (relative to z-axis) given by $\tan \theta_{m}=|\partial \varphi / \partial r| /|\partial \varphi / \partial z|$, where $\partial \varphi / \partial z=k-\left(1-f_{\varphi}\right) Z_{R}^{-1}\left(1+\alpha^{2}\right)^{-1}$, $\partial \varphi / \partial r=k r \alpha Z_{R}^{-1}\left(1+\alpha^{2}\right)^{-1}, f_{\varphi}=r^{2}\left(1-\alpha^{2}\right) w_{0}^{-2}\left(1+\alpha^{2}\right)^{-2}$. From these equations, it is straightforward to find the subluminous phase velocity regions. The condition $v_{\varphi z}<c$ requires $f_{\varphi}>1$, which can only occur in the region $z<Z_{R}$. Moreover, at $z=0, f_{\varphi}>1$ occurs only for $r>w_{0}$. As for the minimum phase velocity, the condition $v_{\varphi m}<c$ requires approximately $r>w(z)$, which extends to a region much larger than that for $v_{\varphi z}<c$. In the subluminous phase velocity region, the magnitude of the minimum phase velocity is of the order $v_{\varphi m} \sim c\left[1-1 /\left(k w_{0}\right)^{2}\right]$, and $\theta_{m} \sim 1 /\left(k w_{0}\right)$. The distribution of $v_{\varphi m}$ on the plane $y=0$ is shown in Fig. 1.

Notice that the effective phase velocity along the z-axis is superluminous, i.e., $\left(v_{\varphi z}\right)_{r=0}=c /\left\{1-1 /\left[k Z_{R}\left(1+\alpha^{2}\right)\right]\right\}$. This indicates that the near-axis region of the beam is not suitable for accelerating charged particles. This is because the phase velocity in this region is the highest, which leads to fast phase slippage. Also, in the near-axis region the longitudinal component of the accelerating electric field is very small.

For accelerating particles, in addition to the subluminous phase velocity of the field, the acceleration field strength, i.e., the amplitude of the longitudinal electric field, is also an important factor. We hereby introduce a quantity $Q$ that combine these two factors together to represent the ability of the laser field for accelerating charged particles. We call it acceleration quality factor, which is defined by

$$
Q=Q_{0}\left(1-v_{\varphi m} / c\right)[x / w(z)] \exp \left[-\left(x^{2}+y^{2}\right) / w(z)^{2}\right]
$$


for $v_{\varphi m} \leq c$ and $Q=0$ for $v_{\varphi m}>c$. Here $Q_{0}$ is a normalization constant chosen to make $Q$ in the order of unity. In Eq. (7), $\left(1-v_{\varphi m} / c\right)$ represents the contribution from the phase velocity, and the remaining factor is proportional to the amplitude of the longitudinal electric field. Also, we simply assume that for $v_{\varphi m}>c, Q \equiv 0$ because in that case no particle can remain trapped in (i.e., synchronous with) the accelerating phase region of the laser field.

The distribution of the acceleration quality factor $Q$ on the plane $y=0$ for a focused laser beam with $k w_{0}=60$ is given in Fig. 2. It is apparent, judging from Fig. 2, that there is an acceleration channel in the field of the focused laser beam propagating in vacuum, which shows similar characteristics to that of a wave guide tube of conventional accelerators: a subluminous phase velocity in conjunction with a strong longitudinal electric field component. Consequently, if one can inject fast electrons into this channel, then these electrons can remain synchronous with the accelerating phase for sufficiently long times such that they receive considerable energy from the field.

To study the detailed dynamics of electrons in this laser acceleration channel, 3D test particle simulations are utilized that solve the relativistic Newton-Lorentz equations of motion, $d \mathbf{P} / d t=-e(\mathbf{E}+\mathbf{v} \times \mathbf{B})$, where $\mathbf{v}$ is the electron velocity in units of $c, \mathbf{P}=\gamma \mathbf{v}$ is the electron momentum in units of $m c$, and $\gamma=\left(1-\mathbf{v}^{2}\right)^{-1 / 2}$ the Lorentz factor. To describe the laser field, in addition to $E_{x}$ given by Eq.(1), the other electric and magnetic components are obtained by solving analytically (to leading order in $1 / k Z_{R}$ ) Maxwell's equations in vacuum [15], $E_{z} \simeq(i / k)\left(\partial E_{x} / \partial x\right)$ and $\mathbf{B} \simeq-(i / \omega) \nabla \times \mathbf{E}$.

Results of these simulations indicate that there exists a regime in which relativistic electrons can be injected into the acceleration channel of a very intense laser beam and receive substantial energy gains. We call this the CAS $[12,13]$. The basic conditions 
for CAS to occur have been found as follows. (i) The laser intensity should be very strong $\left(a_{0} \gtrsim 5\right)$. (ii) The electron injection energy should be in the range $5-15 \mathrm{MeV}$, depending on the beam width. (iii) The electron incident crossing angle (relative to the beam direction) should be small (typically $\tan \theta \sim 0.1$ ). Figure 3 shows typical CAS trajectories at different laser intensity. These CAS trajectories have in common the following interesting dynamic characteristics. (i) The CAS electrons are trapped in and move along the acceleration channel, which are different from the ponderomotively scattered (PS) electrons. The later are quickly expelled from the intense field region (Fig.3(a)). (ii) The effective wave phase velocity along the CAS electron trajectories are less than $\mathrm{c}$ in the region near the beam waist (can even be less than the particle velocity, see Fig.3(e)). (iii) The CAS electrons are phase synchronous with the laser field over a significant distance (Fig.3(d)), which results in substantial net energy gains from the laser field (Fig.3(b)). The acceleration occurs primarily in the focus region with effective interaction lengths on the order of the Rayleigh range (Fig.3(c)) and acceleration gradients on the order of a few $\mathrm{GeV} / \mathrm{cm}$ (Fig.3(b)).

Figure 4 shows the variation of $\gamma_{f m}$ as a function of the laser intensity $a_{0}$. Here, $\gamma_{f}$ is the final value of $\gamma$ after the interaction, and $\gamma_{f m}$ denotes the maximum value of $\gamma_{f}$ as the initial laser phase $\varphi_{0}$ is varied over the range 0 to $2 \pi$. Various values of $\varphi_{0}$ correspond to electrons impinging on the laser beam at different delay time. We note the dynamic trajectories in Fig. 4 can generally be divided into two groups: CAS for $a_{0} \gtrsim 8$, and PS electrons for $a_{0} \lesssim 3$. The CAS electrons are greatly accelerated by the laser field, and the net energy gain scales approximately as $\gamma_{f m} \propto a_{0}^{n}$ with $n \sim 1$. This feature is consistent with the mechanism underlying CAS, namely the acceleration occurs primarily in the acceleration channel. In contrast to that, the PS electrons have only small amount 
of net energy exchange with the laser field [12,13].

The above simulation results are in approximate agreement with the following simple theory of laser acceleration. The energy gain is given by $W=-e \int d t(\mathbf{v} \cdot \mathbf{E})$. Assuming a small electron trajectory angle, $\theta \simeq\left|v_{x} / v_{z}\right| \ll\left|E_{z} / E_{x}\right| \sim\left(x / Z_{R}\right)\left(w_{0}^{2} / w^{2}\right)$, i.e., small compared to the diffraction angle (see, e.g., Fig.3(a)), then the energy gain is primarily due to the axial electric field $E_{z} \simeq\left(2 i x / k w^{2}\right)\left(1+i z / Z_{R}\right) E_{x}$. In this case

$$
W=-m c^{2} a_{0} k w_{0} \int \frac{d z}{Z_{R}} \frac{x w_{0}^{2}}{w^{3}}\left(i-\frac{z}{Z_{R}}\right) \exp \left(-\frac{r^{2}}{w^{2}}-i \varphi\right) .
$$

The magnitude of the energy gain can be estimated by assuming that the effective acceleration length is a Rayleigh length and by approximating $w$ and $\varphi$ as constants. This gives $W[\mathrm{MeV}] \simeq 0.51 a_{0} k w_{0} x \exp \left(-r^{2} / w_{0}^{2}\right)$. Assuming $x \simeq r \simeq w_{0}$ gives $W[\mathrm{MeV}] \simeq$ $0.19 a_{0} k w_{0}=8.1(P[\mathrm{TW}])^{1 / 2}$, where $P=0.54\left(a_{0} k w_{0}\right)^{2} \mathrm{GW}$ is the laser power. For the parameters of Fig. $4\left(k w_{0}=60\right), W \simeq 110 \mathrm{MeV}(1.1 \mathrm{GeV})$ for $a_{0}=10(100)$, in approximate agreement with those from the simulation $W \simeq 100 \mathrm{MeV}(1.5 \mathrm{GeV})$. A more accurate calculation of the energy gain can be obtained by performing the integration in Eq. (5) numerically using the exact orbits, including effects due to the difference between the electron and phase velocities, as well as contributions from the transverse laser field.

In the case of injecting an electron bunch of duration long compared to the laser pulse duration, the output of the acceleration mechanism is a high-energy electron macro-pulse, which consists of many micro-pulses. The macro-pulse duration is that of the laser pulse, and the micro-pulse duration corresponds to the periodicity of the laser field. The energy spread of the electrons is large, since the electrons are injected over all phases within a single laser period, thus some electrons are accelerated and others ponderomotively scattered, depending on the phase when the electron impinges on the laser field. The 
CAS electrons undergoing favorable acceleration can be as high as $30 \%$ of the incident electrons intersecting with the laser pulse.

Following are two examples that show the output beam properties in quantitative detail. An electron bunch was synchronously injected to interact with a laser pulse with $k w_{0}=80$, duration $\omega \tau=300$, and $a_{0}=10$ (and 30). Initially, the bunch had momenta $p_{x i}=1.2, p_{y i}=0, p_{z i}=12,\left(\operatorname{crossing}\right.$ angle $\left.\vartheta=\tan ^{-1}(0.1)\right)$, emittance $0.1 \pi \mathrm{mm}-\mathrm{mrad}$, and impact parameter $b=0$, and a transverse size equal to that of the laser pulse. After the interaction, the maximum energy was $130 \mathrm{MeV}(625 \mathrm{MeV})$, and $8 \%(21 \%)$ of the injected electrons had energies above $80 \mathrm{MeV}(400 \mathrm{MeV})$. Futhermore, there is an energyangle correlation that can allow for energy selection using a collimator. For electrons in the energy range $104 \mathrm{MeV} \pm 15 \%$ (500 $\mathrm{MeV} \pm 15 \%$ ), which contained $22 \%$ (37.6\%) of the accelerated electrons and emerged with angles $\theta=2^{\circ} \pm 0.16^{\circ},\left(0.99^{\circ} \pm 0.15^{\circ}\right)$, the emittances (mm-mrad) in the $\mathrm{x}$ and y directions were $0.046(0.037)$ and $0.37(0.45)$, respectively. Details on these simulations will be a subject of a future publication.

In summary, we report a new regime of laser acceleration in vacuum in which the energy gain is found to increase approximately linear in $a_{0}$, and in which substantial energy gains $(>100 \mathrm{MeV})$ can be obtained for present day lasers ( $\gtrsim 100 \mathrm{TW})$. Finite energy gains are possible because (i) the electron orbit is significantly perturbed by the laser as it enters the high intensity region, and (ii) there exists a subluminous phase velocity region in which the electrons can become captured and accelerated. The resulting energy gains are in agreement with theoretical estimates based on acceleration in the axial laser field. Furthermore, optical components are not needed near the laser focus, which greatly simplifies the experimental interaction geometry and removes constraints due to laser damage, thus allowing ultra-high intensities and large energy gains. 
The authors would like to thank C.M. Fou for a careful reading of this manuscript. This work was partly supported by the National Natural Science Foundation of China under Contracts No. 19984001 and No. 10076002, National High-Tech ICF Committee in China, Engineering - Physics Research Institute Foundation of China, and National Key Basic Research Special Foundation (NKBRSF) under grant No. G1999075200. E.E. and A.S. are supported by the U.S. Department of Energy, Office of Science, Contract No. DE-AC03-76SF00098. 


\section{References \& Notes}

* Author to whom correspondence should be addressed. Fax: +86-21-6564-3815, Email: hoyk@fudan.ac.cn

[1] M.D. Perry, and G.A. Mourou, Science 264, 917 (1994); G.A. Mourou, C.P.J. Barty, and M.D. Perry, Phys. Today 51(No.1), 22 (1998).

[2] M. Everett, et al., Nature 368, 527 (1994); A. Modena, et al., Nature 377, 606 (1995).

[3] D. Umstadter, S.Y. Chen, A. Maksimchuk, G. Mourou, and R. Wagner, Science 273, 472 (1996); D. Umstadter, Phys. Plasmas 8, 1774 (2001).

[4] E. Esarey, P. Sprangle, J. Krall, and A. Ting, IEEE Trans. Plasma Sci. 24, 252 (1996); W.P. Leemans et al., Phys. Plasmas 8, 2510 (2001).

[5] K. Nakajima, et al., Phys. Rev. Lett. 74, 4428 (1995); C.I. Moore, et al., Phys. Rev. Lett. 79, 3909 (1997); F. Amiranoff, et al., Phys. Rev. Lett. 81, 995 (1998); K. Krushelnick, et al., Phys. Rev. Lett. 83, 737 (1999); C. Gahn, et al., Phys. Rev. Lett. 83, 4772 (1999).

[6] P.M. Woodward, J. IEE 93, 1554 (1947); J.D. Lawson, IEEE Trans. Nucl. Sci. NS-26, 4217 (1979); R.B. Palmer, Part. Accel. 11, 81 (1980).

[7] E. Esarey, P. Sprangle, and J. Krall, Phys. Rev. E 52, 5443 (1995); P. Sprangle, E. Esarey, and J. Krall, Phys. Plasmas 3, 2183 (1996).

[8] G. Malka, E. Lefebvre, and J.L. Miquel, Phys. Rev. Lett. 78, 3314 (1997); K.T. McDonald, Phys. Rev. Lett. 80, 1350 (1998): P. Mora, and B. Quesnel, Phys. 
Rev. Lett. 80, 1351 (1998); E. Lefebvre, G. Malka, and J.L. Miquel, Phys. Rev. Lett. 80, 1352 (1998).

[9] B. Quesnel, and P. Mora, Phys. Rev. E58, 3719 (1998).

[10] F.V. Hartemann, et al., Phys. Rev. E 58, 5001 (1998).

[11] G.V. Stupakov, and M.S. Zolotorev, Phys. Rev. Lett. 86, 5274 (2001).

[12] J.X. Wang, et al., Phys. Rev. E 58, 6575 (1998); L.J. Zhu, et al., Phys. Lett. A 248, 319 (1998); Q. Kong, et al., Phys. Rev. E 61, 1981 (2000).

[13] P.X. Wang, Y.K. Ho, X.Q. Yuan, Q. Kong, N. Cao, A.M. Sessler, E. Esarey, and Y. Nishida, Appl. Phys. Lett. 78, 2253 (2001).

[14] Y.C. Huang, R.L. Byer, Appl. Phys. Lett. 69, 2175 (1996); R.L. Byer, T. Plettner, C. Barnes, E. Colby, B. Cowan, R.H. Siemann, J.E. Spencer, Proc. 2001 Particle Accel. Conf. (in press).

[15] The E-M field given here is the analytical expression in the paraxial approximation for continuous Gaussian beams. It should be pointed out that the results given in this article are found to be correct even if the high-order corrections to the paraxial expressions are taken into account. 


\section{Captions}

Fig. 1: The distribution of the minimum phase velocity $v_{\varphi m}$ in the $y=0$ plane of a focused laser beam with $k w_{0}=60$. The value $v_{\varphi m}$ is given in units of $c . \beta=x / w_{0}$ and $\alpha=z / Z_{R}$

Fig. 2: The acceleration quality factor (Eq. (7)) for a focused laser beam of $k w_{0}=60$ in the plane of $y=0$. Again, $\beta=x / w_{0}$ and $\alpha=z / Z_{R}$.

Fig. 3: Typical CAS dynamic trajectories at different laser intensities. (a) CAS dynamic trajectories in the x-z coordinate space for $a_{0}=100$ (solid line), 60 (dashed line), and 30 (fine-dotted line). The other parameters used are $k w_{0}=60, P_{x i}=1.2$, $P_{y i}=0, P_{z i}=12.0$. In the figure, the dash-dotted line shows the beam width $w(z)$. As a contrast, three typical ponderomotively scattered electron trajectories corresponding to the same parameters as that of the CAS's are also presented. (b) Same parameters as in (a) but for $\gamma$ vs. z-coordinate. (c) Same parameters as in (a) but for the longitudinal force experienced by the electrons vs. z-coordinate. (d) Same parameters as in (a) but for the phase experienced by the CAS electrons vs. z-coordinate. (e) Same parameters as in (a) but for the electron velocity (solid line) and the phase velocity of the laser wave along the electron dynamic trajectory (dotted line) vs. z-coordinate at $a_{0}=30$.

Fig. 4: The maximum final energy $\gamma_{f m}$ as a function of the laser intensity $a_{0} \cdot \gamma_{f m}$ denotes the maximum value of $\gamma_{f}$ as the initial laser phase $\varphi_{0}$ is varied over the range 0 to $2 \pi$. The other parameters used are the same as in Fig. 3. 
Fig.1

Y.K. Ho et al.

PRL

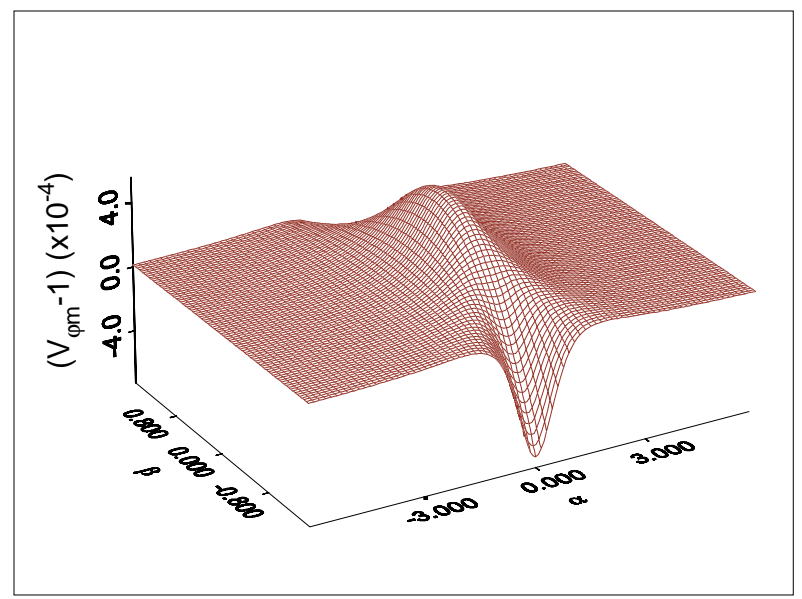

Fig. 1 
Fig.2

Y.K. Ho et al.

PRL

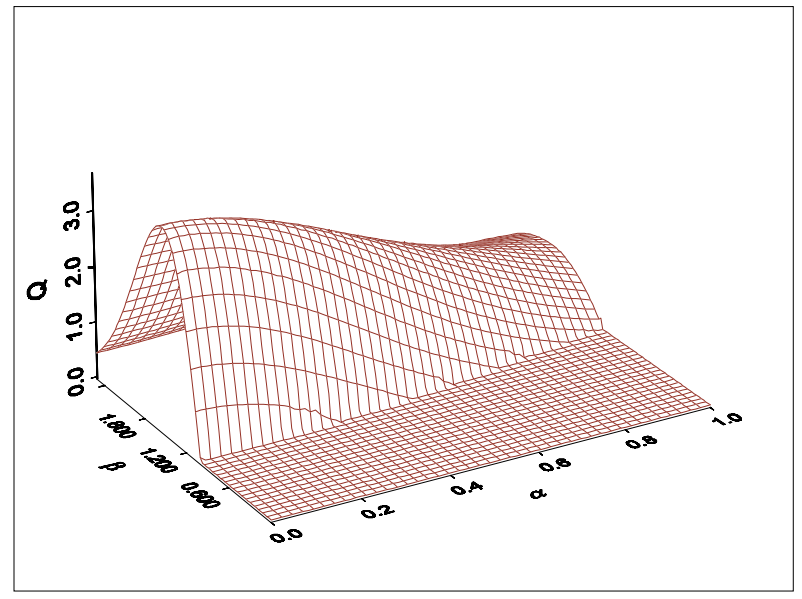

Fig. 2 
Fig.3

Y.K.Ho et al.

PRL
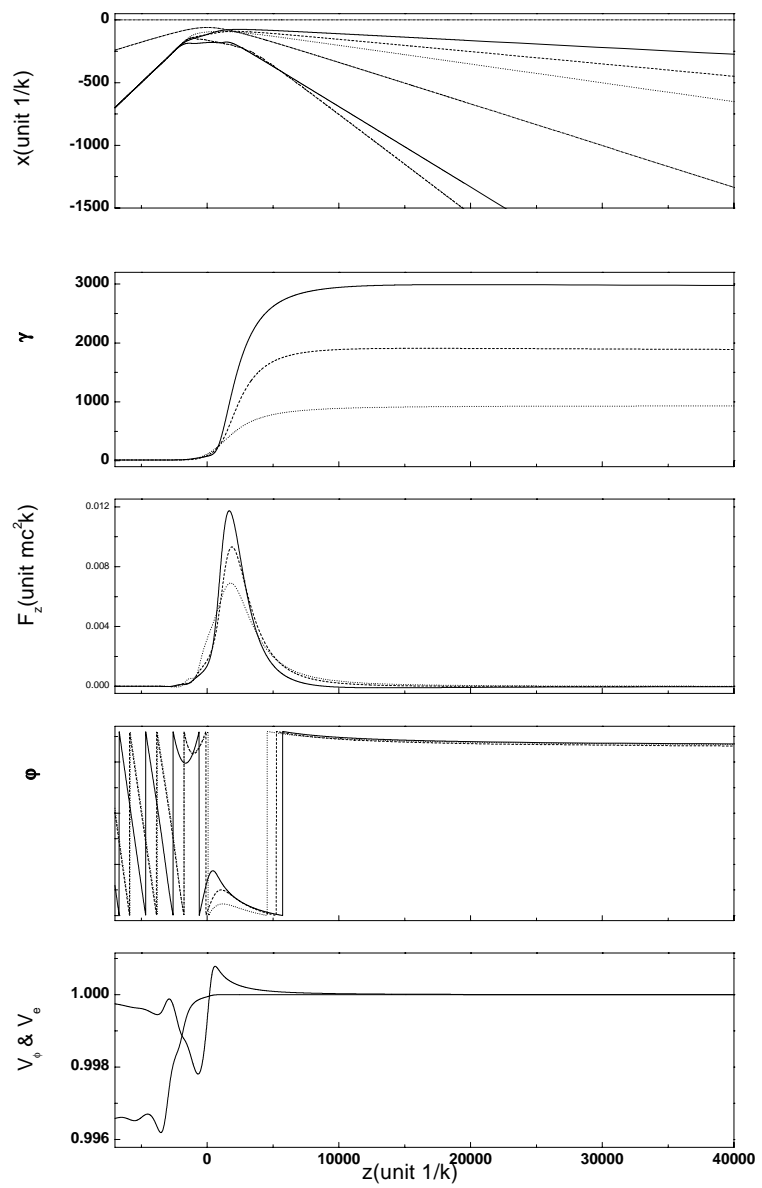

Fig. 3 
Fig. 4

Y.K. Ho et al.

PRL

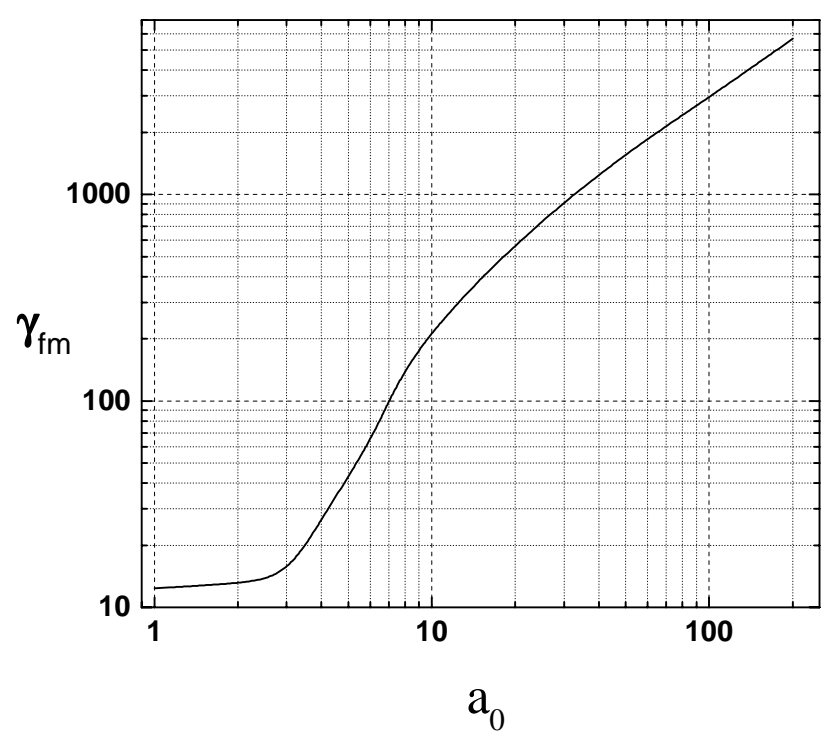

Fig. 4 